\title{
Atividade predatória do fungo Monacrosporium thaumasium contra o nematóide Haemonchus contortus, após passagem pelo trato gastrintestinal de caprinos
}

\author{
Predatory activity of nematophagous fungus Monascroporium \\ thaumasium against Haemonchus contortus, after passage \\ through the gastrointestinal \\ tract of goats
}

\section{Luciana Magalhães Melo' Claudia Maria Leal Bevilaqua² Jackson Victor de Araújo ${ }^{3}$ Ana Carolina Fonseca Lindoso Melo ${ }^{4}$.}

\section{-NOTA-}

\section{RESUMO}

Conidios de Monacrosporium thaumasium foram administrados por via oral a caprinos para verificar a viabilidade desse fungo nematófago após passagem pelo trato gastrintestinal. Foram realizadas amostragens de fezes de 18 a 30 horas após inoculação. Constatou-se crescimento do fungo e atividade predatória contra Panagrellus spp em placas confeccionadas com fezes coletadas 21 e 24 horas após a inoculação. Houve uma redução média de 79,24\% no número de larvas infectantes de Haemonchus contortus provenientes de coproculturas preparadas com fezes coletadas 24 horas após a administração do fungo. O fungo M. thaumasium manteve atividade predatória sobre $\boldsymbol{H}$. contortus, após passagem através do trato gastrintestinal de caprinos.

Palavras-chave: caprino, controle biológico, fungo nematófago, Monacrosporium thaumasium, Haemonchus contortus, Panagrellus spp.

\section{ABSTRACT}

In order to verify the viability after passage through the gastrointestinal tract, conidia of nematophagous fungus,
Monacrosporium thaumasium, were orally administered to goats. Fecal samples were collected 18 to 30 hours after inoculation. It was verified fungus growth and predatory activity against Panagrellus spp in plates made with feces collected at 21 and 24 hours after inoculation. There was a 79.24\% reduction of Haemonchus contortus infective larvae number from coprocultures prepared with feces collected at 24 hours after the fungus administration. The fungus M. thaumasium maintained predatory activity over $\boldsymbol{H}$. contortus, after passage through the gastrointestinal tract of goat.

Key words: goat, biological control, nematophagous fungus, Monacrosporium thaumasium, Haemonchus contortus, Panagrellus spp.

O parasitismo por nematóides gastrintestinais limita o desenvolvimento da caprinocultura, sendo Haemonchus contortus o mais importante parasito (AROSEMENA et al., 1999). O aparecimento da resistência anti-helmíntica impulsionou pesquisas sobre controle biológico desses parasitos usando fungos nematófagos. Para que esses fungos possam ser administrados aos animais em épocas

\footnotetext{
${ }^{1}$ Acadêmico de Medicina Veterinária, Faculdade deVeterinária , Universidade Estadual do Ceará (UECE), Bolsista PIBIC / CNPq. ${ }^{2}$ Professor adjunto FAVET, UECE, Avenida Paranjana, 1700, Fortaleza - CE, 60.740-000.

Autor para correspondência. E-mail: claudiam@fortalnet.com.br

${ }^{3}$ Professor adjunto, Universidade Federal de Viçosa.

${ }^{4}$ Mestrando do Programa de Pós-graduação em Ciências Veterinárias, FAVET, UECE.
} Recebido para publicação18.06.01 Aprovado em 20.03.02 
estratégicas, devem sobreviver à passagem pelo trato gastrintestinal (TGI) do animal e manter a atividade predatória nas fezes (ARAÚJO et al., 1999). No presente estudo, os objetivos foram: verificar se o fungo nematófago Monacrosporium thaumasium sobrevive à passagem pelo TGI de caprinos, e comprovar a manutenção da atividade predatória sobre larvas infectantes de $\boldsymbol{H}$. contortus após essa passagem.

Conídios de M. thaumasium (isolado NF34A) foram obtidos, estocados e quantificados segundo a técnica descrita por ARAÚJO (1996). Panagrellus spp, nematóides de vida-livre, foram cultivados e limpos de acordo com ARAÚJO (1996).

Três caprinos adultos, previamente vermifugados com ivermectina $(200 \mathrm{mg} / \mathrm{mL})$, receberam $9 \times 10^{6}$ conídios, divididos em três doses iguais, intervaladas em uma hora, formando o grupo tratado. Outros três caprinos serviram de controle para o experimento. Desde cinco dias antes da inoculação do fungo até o final do experimento, os animais foram arraçoados com concentrado de milho e capim autoclavados. Amostras de fezes foram coletadas diretamente da ampola retal de cada animal a 18,21, 24,27 e 30 horas após a primeira administração do fungo.

Para visualização do fungo nas fezes coletadas, foram utilizadas as amostras provenientes de um animal do grupo tratado e de um do grupo controle. Cada amostra foi homogeneizada e dividida em 3 partes de 2 gramas e cada parte foi distribuída na região central de uma placa de Petri com ágar-água a $2 \%$. Essas placas foram adicionadas de 2.000 Panagrellus spp, a cada 2 ou 3 dias, e incubadas a temperatura ambiente e no escuro, sendo observadas tras provenientes de dois animais do grupo tratado e dois do grupo controle. Cada amostra foi homogeneizada, originando duas coproculturas, de acordo com o seguinte protocolo: um a três gramas de fezes do animal foram misturadas a dois gramas de fezes contendo aproximadamente 1.200 ovos de $\boldsymbol{H}$. contortus, provenientes de animal com infecção pura. Após homogeneização, as culturas foram incubadas por 18 dias à temperatura ambiente e no escuro. As larvas foram recuperadas pela técnica de Roberts \& O'Sullivan descrita por UENO \& GUTIERRES (1998), estocadas a $4^{\circ} \mathrm{C}$ e, posteriormente, quantificadas. $\mathrm{O}$ número de larvas foi transformado pela fórmula $\log (\mathrm{x}+0,5)$, onde $\mathrm{x}=$ número de larvas recuperadas de cada coprocultura. As médias dos valores transformados foram submetidas à análise de variância (ANOVA) e comparadas pelo teste de Tukey $(\mathrm{p}<0,05)$.

A presença de fungo nematófago com características morfológicas compatíveis com $\boldsymbol{M}$. thaumasium foi constatada apenas nas placas confeccionadas com fezes do grupo tratado, coletadas às 21 e 24 horas após a administração dos conídios, sugerindo que a passagem dos conídios pelo TGI do caprino ocorreu nesse intervalo de tempo. Nessas placas, foram visualizados Panagrellus spp predados, indicando a manutenção da atividade predatória do fungo sobre esse nematóide. O número de larvas recuperadas do grupo tratado foi diferente do controle somente às 24 horas após administração do fungo (Tabela 1), havendo uma redução média de $79,24 \%$. Isso sugere que M. thaumasium manteve atividade predatória sobre H. contortus, após atravessar o TGI de caprinos. Esse fungo demonstrou semelhante desempenho sobre lar-

Tabela 1 - Média do número de larvas infectantes de Haemonchus contortus recuperadas de coproculturas confeccionadas com fezes de caprinos coletadas após a administração oral de conídios de Monacrosporium thaumasium.

\begin{tabular}{|c|c|c|c|c|c|}
\hline \multirow[b]{2}{*}{ Grupo } & \multicolumn{5}{|c|}{ Média de larvas ( \pm desvio padrão) em diferentes momentos pós-inoculação (horas) } \\
\hline & 18 & 21 & 24 & 27 & 30 \\
\hline Tratado & $57,5 \pm 61,8$ & $16,2 \pm 26,3$ & $5,5 \pm 11,0^{\mathrm{a}}$ & $24,5 \pm 16,9$ & $13,5 \pm 13,9$ \\
\hline Controle & $66,0 \pm 65,2$ & $69,7 \pm 52,6$ & $26,5 \pm 4,1^{\mathrm{b}}$ & $15,0 \pm 23,8$ & $44,0 \pm 50,0$ \\
\hline
\end{tabular}

Letras diferentes indicam diferença significativa $(\mathrm{p}<0,05)$.

diariamente, por 12 dias, sob estereomicroscópio (40x a 160x). A identificação das características morfológicas de $\boldsymbol{M}$. thaumasium foi realizada com base na chave proposta por LIU \& ZHANG (1994).

Para verificar a manutenção da atividade nematófaga sobre $\boldsymbol{H}$. contortus, foram utilizadas amos- vas de Haemonchus placei após passagem pelo trato de bovinos (ARAÚJO et al., 1999). A tendência à diminuição do número de larvas às 21 horas indica que o fungo esteve presente nas coproculturas desse horário, contudo, em quantidade insuficiente para produzir o efeito observado às 24 horas. 
Não existe, na literatura disponível, trabalhos específicos investigando a sobrevivência de fungos nematófagos após passagem pelo TGI de caprinos.

Estudos com animais em condições naturais de pastejo são escassos e apresentam pouco sucesso (LARSEN et al., 1994; MANUELI et al., 1999).

Os resultados do presente estudo abrem perspectivas para a utilização de $\boldsymbol{M}$. thaumasium como bioagente no controle de $\boldsymbol{H}$. contortus, sobretudo em criações de caprinos. No entanto, ainda são necessários estudos da eficácia do fungo a campo.

\section{REFERÊNCIAS BIBLIOGRÁFICAS}

ARAÚJO, J.V. Interação entre as larvas infectantes de Cooperia punctata e os fungos predadores do gênero Arthrobotrys, caracterização dos isolados de Arthrobotrys e seu uso no controle biológico de nematóides gastrintestinais de bovinos. 1996. 110f. Tese (Doutorado) - Instituto de Ciências Biológicas, Universidade Federal de Minas Gerais.

ARAÚJO, J.V.; STEPHANO, M.A.; SAMPAIO, W.M. Passage of nematode-trapping fungi through the gastrointestinal tract of calves. Veterinary Archiv, v.69, p.69-78, 1999.
AROSEMENA, N.A.E. et al. Seasonal variations of gastrointestinal nematodes in sheep and goats from semi-arid area in Brazil. Revue de Médicine Véterinaire, v.150, p.873876, 1999

LARSEN, M.; FAEDO, M.; WALLER, P.J. The potential of nematophagous fungi to control the free-living stages of nematode parasites of sheep: survey for the presence of fungi in fresh faeces of grazing livestock in Australia. Veterinary Parasitology, v.53, p.275-281, 1994.

LIU, X.Z.; ZHANG, K.Q. Nematode-trapping species of Monacrosporium with special reference to two new species. Mycological Research, v.98, p.862-868, 1994.

MANUELI, P.R. et al. Biological control of nematode parasites of livestock in Fiji: screening of fresh dung of small ruminants for the presence of nematophagous fungi. Veterinary Parasitology, v.81, p.39-45, 1999.

UENO, H.; GUTIERRES, P.C. Manual para diagnóstico das helmintoses para ruminantes. Tokyo : Japan International Cooperation Agency, 1998. 143p. 